\title{
The effectiveness of internal compression therapy in deep venous insufficiency
}

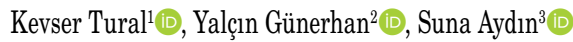 \\ ${ }^{1}$ Department of Cardiovascular Surgery, Kafkas University, School of Medicine, Kars, Turkey \\ ${ }^{2}$ Department of Cardiovascular Surgery, Harakani State Hospital, Kars, Turkey \\ ${ }^{3}$ Department of Cardiovascular Surgery, Health Science University, Fethi Sekin City Hospital, Elazığ, Turkey
}

Received: August 21, 2020 Accepted: September 29, 2020 Published online: October 21, 2020

\begin{abstract}
Objectives: The aim of this study was to evaluate the effectiveness of internal compression therapy (ICT) in patients with primary deep venous insufficiency (DVI).

Patients and methods: A total of 13 extremities of 11 patients ( 4 males, 7 females; mean age $54.3 \pm 13.7$ years; range, 34 to 76 years) who were diagnosed with primary lower extremity deep venous reflux and underwent ICT for symptomatic DVI between January 2018 and December 2018 were included in this retrospective study. The Venous Clinical Severity Score (VCSS) was performed in all patients in the pre- and postoperative period to assess symptomatic improvements. Control examinations of the patients were performed on post-procedural Day 3 and at 1, 6, and 12 months.

Results: The mean diameter of deep veins was $13.0 \pm 1.8$ (range, 10.2 to 16.1 ) $\mathrm{mm}$ and the mean distance between the valves was $3.9 \pm 1.2$ (range, 2.1 to 6.1 ) $\mathrm{mm}$ in the pre-procedural period. The mean reflux time was $3.3 \pm 0.5$ (range, 2.5 to 4 ) min. The mean diameter of deep veins was $8.9 \pm 1.2$ (range, 7.5 to 11.0 ) $\mathrm{mm}$ at 12 months after the procedure. The success of the treatment was $100 \%$ after the treatment and on the post-procedural Day 3. The mean VCSS of the patients was $8.1 \pm 3.1$ (range, 6.0 to 16.0 ) in the pre-procedural period and $1.1 \pm 1.4$ (range, 0.0 to 4.0$)$ at 12 months during follow-up ( $\mathrm{p}=0.001$ ).

Conclusion: The ICT appears to be a promising procedure, as it is a minimally invasive, rapid, and effective method for the treatment of patients with DVI.
\end{abstract}

Keywords: Femoral vein, internal compression therapy, venous insufficiency, venous valves, therapeutics.

Deep venous insufficiency (DVI) is a major public health problem and its incidence increases with aging, female sex, previous deep venous thrombosis (DVT) history, pregnancy, obesity, and smoking. ${ }^{[1]}$ It can occur due to primary valvular insufficiency, congenital valve malformations, or valve malfunction secondary to DVT. ${ }^{[2]}$ Deep venous insufficiency leads to the loss of labor force and health costs by causing infection, extreme swelling, soft tissue ulceration, and DVT. ${ }^{[3]}$ The treatment of DVI is important in decreasing complaints such as pain, swelling, skin pigmentation, and venous ulcer. ${ }^{[4,5]}$ Compression therapy, despite significant improvements of other methods, still remains the cornerstone of conservative treatment. ${ }^{[1]}$ Surgical treatments including venous valvular repair, transposition of vein, neovalve construction, and external valve banding require local or general anesthesia. ${ }^{[4,5]}$ The success of valve reconstruction surgery in the treatment of primary DVI is about 65 to $70 \%$ during five-year follow-up..$^{[6,7]}$ Due to their less successful results and impracticality, such surgery procedures are not performed as routine procedures in many centers..$^{[4,6-9]}$

Internal compression therapy (ICT) with a paravalvular leak device (Invamed, Ankara, Turkey) is a new treatment method for primary DVI (Figure 1). The paravalvular leak device is a device which provides to be delivered a mixture of hyaluronic acid and n-butyl-2-cyanoacrilate (NBCA) hard gel on the outer surface of the vein in the insufficient valve level between the deep vein and muscle fascia. The mixture of hyaluronic acid and NBCA adheres around the valves and forms a granulomatous structure. Thus, this device helps to the valves approach each other and

Corresponding author: Kevser Tural, MD. Kafkas Üniversitesi Tıp Fakültesi Kalp ve Damar Cerrahisi Anabilim Dal, 36100 Kars, Türkiye.

Tel: +90 505 - 7683030 e-mail: ktrl2011@hotmail.com

\section{Citation:}

Tural K, Günerhan Y, Aydın S. The effectiveness of internal compression therapy in deep venous insufficiency. Cardiovasc Surg Int 2020;7(3):136-142. 
boost their functioning. ${ }^{[10]}$ In the current literature, there exist very few studies about the results of this treatment method, as it is a fairly new method. In the present study, therefore, we aimed to evaluate the effectiveness of ICT in patients with primary DVI therapy and to present our one-year follow-up results.

\section{PATIENTS AND METHODS}

This retrospective study was conducted at Cardiovascular Surgery Clinics of Kafkas University, Faculty of Medicine Hospital between January 2018 and December 2018. A total of 13 extremities of 11 patients ( 4 males, 7 females; mean age $54.3 \pm 13.7$ years; range, 34 to 76 years) who were diagnosed with primary lower extremity deep venous reflux and underwent ICT treatment for symptomatic DVI were included. Inclusion criteria were as follows: $\geq 18$ years of age, primary DVI and having complete follow-up data available at six months and one year postoperatively. Exclusion criteria were as follows: severe ambulation limitation, known thrombophilia syndrome, postthrombotic etiology, a body mass index of $>35 \mathrm{~kg} / \mathrm{m}^{2}$, a deep venous reflux of $<2 \mathrm{sec}$, and severe comorbidity. A written informed consent was obtained from each patient. The study protocol was approved by the Kafkas University, Faculty of Medicine Ethics Committee (Date: 13/05/2020/80576354-050-99/161). The study was conducted in accordance with the principles of the Declaration of Helsinki.

All physical examinations were performed by a cardiovascular surgeon in an outpatient clinic and color Doppler ultrasound (CDUS) scanning was performed by two specialist radiologists. Control examinations of the patients were performed on the post-procedural Day 3 and at 1, 6, and 12 months. In the CDUS examination, the patients were examined in terms of reflux time, grade of deep, superficial, and perforating venous insufficiency, and DVT. In addition, the diameter of the common femoral vein where ICT was applied and the distance between the valves were measured and recorded for each patient during the Valsalva maneuver, while the valves were fully open in the expiratory phase. Pathological venous reflux was defined as venous incompetence (reflux) during CDUS examination set at retrograde flow longer than $0.5 \mathrm{sec}$ in the superficial venous system, the deep femoral vein, and the calf veins, longer than $1 \mathrm{sec}$ in the common femoral, femoral vein..$^{[1]}$ All patients were evaluated according to the Comprehensive
Classification System for Chronic Venous Disorders (CEAP) classification and Venous Clinical Severity Score (VCSS) classification. Treatment success was defined as reflux-free deep vein valves. A reflux over $0.5 \mathrm{sec}$ was considered as a failure.

\section{Procedural technique}

All ICT procedures were performed under local anesthesia in sterile conditions. The purpose of this procedure is to reduce the diameter of the vein, that is insufficient, and so to reduce the distance between the valves. ${ }^{[10]}$ Therefore, the insufficient valves were found using CDUS (Figure 2). Then, the distance between the insufficient valves and the diameter of the vein were measured at the beginning of the operation. The ICT procedure was performed as previously explained by Yavuz et al. ${ }^{[10]}$ (Figure 3). The first control examination of the patients was performed with CDUS on the post-procedural Day 3 (Figure 4).

\section{Statistical analysis}

Statistical analysis was performed using the IBM SPSS version 22.0 software (IBM Corp., Armonk, NY, USA). Continuous variables were expressed in mean \pm standard deviation (SD) or median (min-max), while categorical variables were expressed in number and percentage. The Wilcoxon signed-rank test was performed to analyze changes in the VCSS between baseline and follow-up. A $p$ value of $<0.05$ was considered statistically significant.

\section{RESULTS}

Baseline demographic and clinical characteristics of the patients are summarized in Table 1 . None of

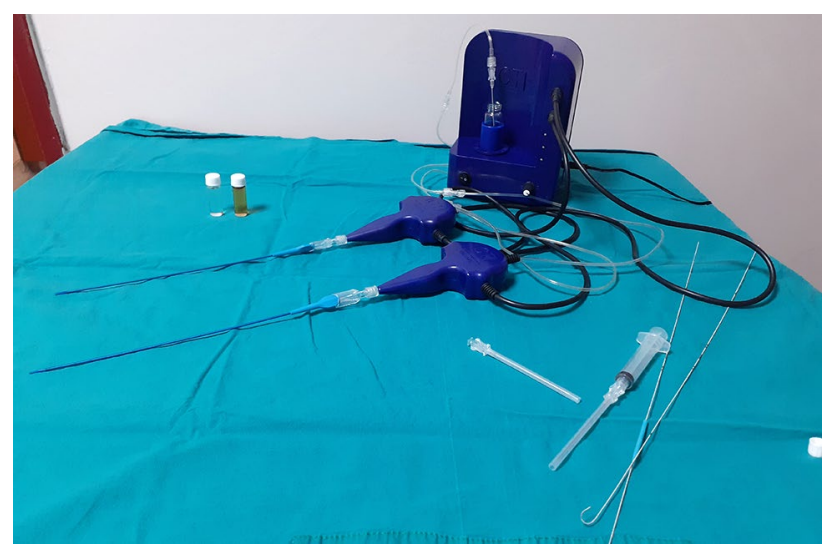

Figure 1. Internal compression therapy Paravalvular Leak Closure Device and Delivery System. 

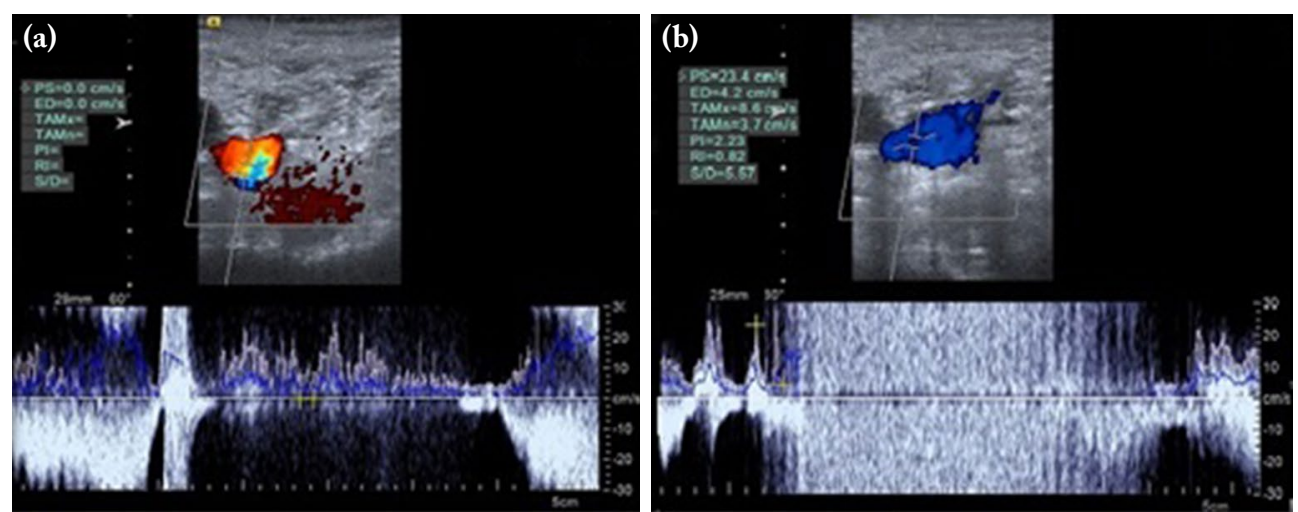

Figure 2. (a) A pre-procedural period image of spontaneous venous flow in right main femoral vein during breathing. (b) A pre-procedural period image of retrograde reflux flow in right common femoral vein with Valsalva maneuver due to deep venous insufficiency.

the patients had a chronic disease such as chronic renal failure, chronic liver failure, heart failure, chronic obstructive pulmonary disease, diabetes mellitus, or DVT. The CEAP classification in the pre-procedural period was $\mathrm{C} 3$ in six lower limbs (46.2\%), C4 in six lower limbs (46.2\%), and C6 in one (7.7\%) lower limb. The mean distance between the valves was $3.9 \pm 1.2$ (range, 2.1 to 6.1 ) $\mathrm{mm}$ in the pre-procedural period. The mean reflux time was $3.3 \pm 0.5$ (range, 2.5 to 4 ) min. The mean procedural time was $22.2 \pm 6.4$ (range, 15 to 35 ) $\mathrm{min}$. The mean delivered amount of ICT hard gel was $3.2 \pm 0.7$ (range, 1.9 to 4.0$) \mathrm{mL}$.

The success of the treatment was $100 \%$ immediately after the treatment and on the post-procedural Day 3 with no reflux observed by CDUS in the deep femoral veins. At the six-month follow-up, the results were similar to the post-procedural Day 3 with vein diameters and reflux-free deep veins. At 12 months of follow-up, no significant reflux was observed over $0.5 \mathrm{sec}$ in deep venous vessels on which ICT was applied.

In three of four patients (Patients 2, 3, and 5) who had insufficiency in the proximal vena saphena magna (VSM) in the pre-procedural period, the deficiency in the proximal VSM completely improved in the post-procedural period. Grade 4 insufficiency in the proximal VSM in one patient (Patient 4) improved to Grade 1-2 in the post-procedural period. Since the proximal VSM diameter of the Patients 3 and 4 expanded to 6.0 and $6.9 \mathrm{~mm}$, respectively, while the ICT tight gel was delivered around the insufficient valves in the common femoral vein simultaneously, it was delivered around the saphenofemoral junction (SFJ). However, in two patients (Patients 6 and 9), there was insufficiency in the VSM, not only proximally, but also throughout the VSM. In Patient 6, the diameter of the saphenous vein was $4 \mathrm{~mm}$ and, in Patient 9, the diameter of the saphenous vein in the left lower extremity was $8.6 \mathrm{~mm}$ at its widest point. In Patient 6, DVI improved in the post-procedural

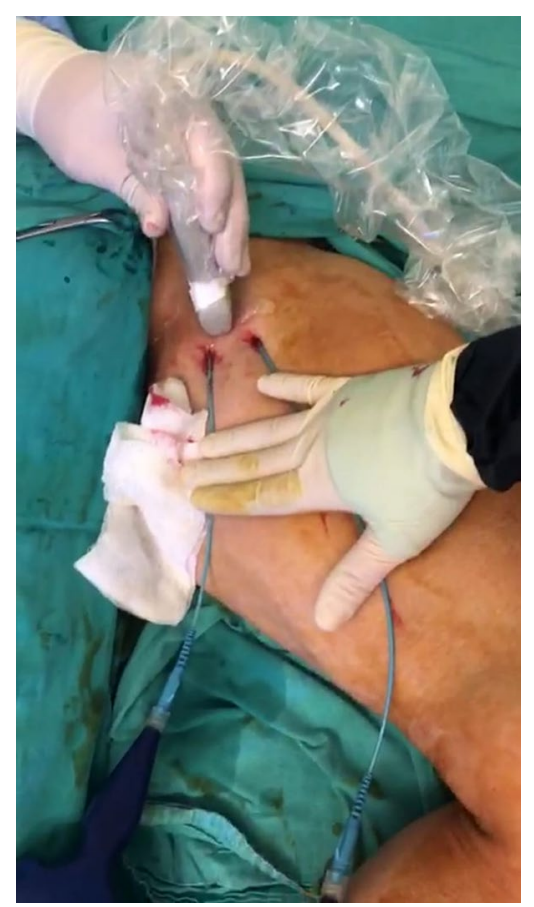

Figure 3. Delivery system cannula placement over to two sides of vein valve. 

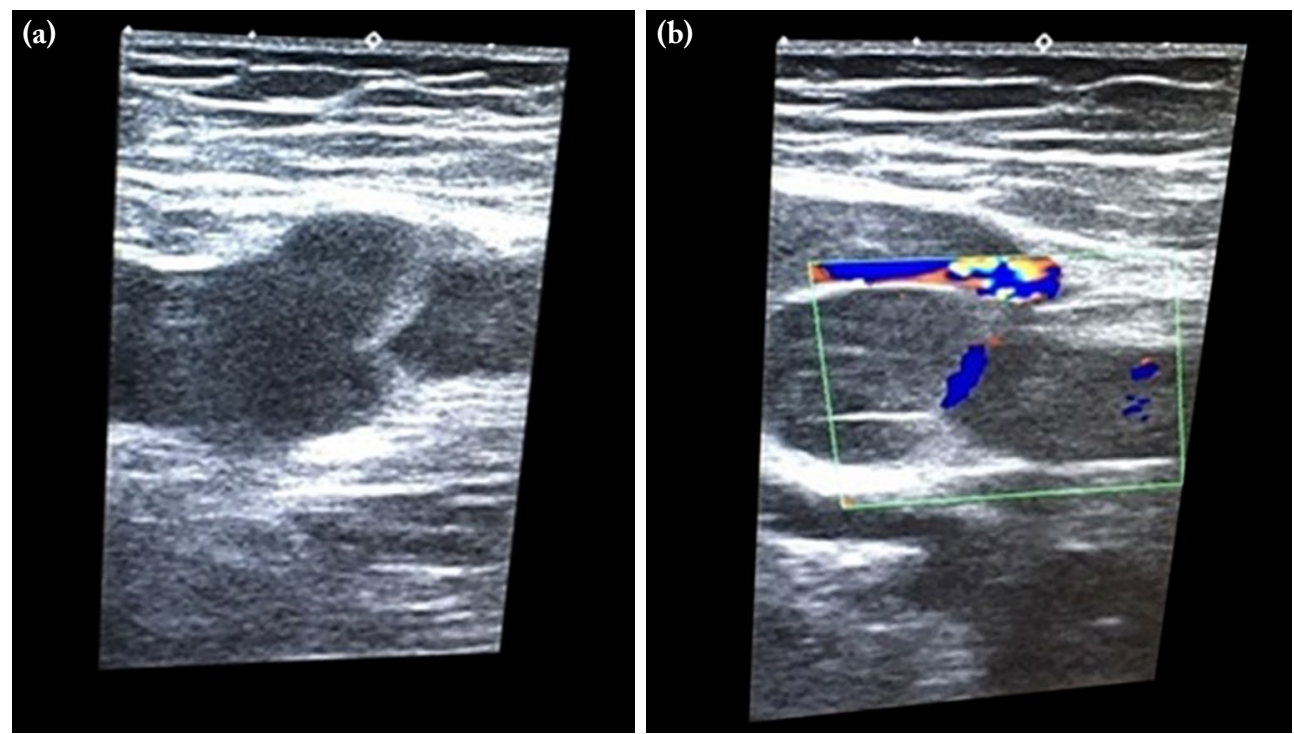

Figure 4. (a) A color Doppler ultrasound image of coaptation of early post-procedural period right main femoral vein valve after internal compression therapy. (b) An image of minimal deep venous insufficiency of right common femoral vein in early post-procedural period during Valsalva maneuver.

period, although the insufficiency in the VSM did not improve due to a long-segment sickness. The use of compression stockings and medical treatment continued regularly in the post-procedural period in this patient whose edema partially improved with the improvement in DVI. Thus, active ulcer regressed at the post-procedural three months of follow-up. In Patient 9, intravenous cyanoacrylate was performed for saphenous vein failure one month before ICT was performed in the left lower extremity.

\begin{tabular}{|c|c|c|c|c|c|c|c|}
\hline & & Baseli & e demographic a & $\begin{array}{l}\text { Table } 1 \\
\text { d clinical characterist }\end{array}$ & s of patients & & \\
\hline Patient/side & Age/Sex & $\begin{array}{c}\text { Deep vein } \\
\text { diameter }(\mathrm{mm})\end{array}$ & $\begin{array}{c}\text { Distance } \\
\text { between valves } \\
(\mathrm{mm})\end{array}$ & $\begin{array}{l}\text { Proximal saphenous } \\
\text { vein insufficient } \\
\text { reflux }(\mathrm{sec})\end{array}$ & $\begin{array}{l}\text { Deep venous } \\
\text { reflux (sec) }\end{array}$ & $\begin{array}{c}\text { CEAP } \\
\text { classification }\end{array}$ & $\begin{array}{l}\text { Pre-procedural } \\
\text { VCSS }\end{array}$ \\
\hline 1 right & $76 / \mathrm{M}$ & 12.4 & 2.1 & - & $2-3$ & 4 & 7 \\
\hline 1 left & & 11.9 & 2.3 & - & $2-3$ & 4 & 7 \\
\hline 2 & $34 / \mathrm{F}$ & 15.2 & 5.7 & $2-3$ & 4 & 3 & 6 \\
\hline 3 & $59 / \mathrm{F}$ & 13.9 & 4.5 & $2-3$ & $3-4$ & 3 & 6 \\
\hline 4 & $63 / \mathrm{M}$ & 12.2 & 3.9 & 4 & 3 & 4 & 7 \\
\hline 5 & $34 / \mathrm{F}$ & 11.5 & 3.5 & 2 & 3 & 3 & 6 \\
\hline 6 & $64 / \mathrm{M}$ & 16.1 & 6.1 & 4 & 3 & 6 & 16 \\
\hline 7 & $60 / \mathrm{M}$ & 13.2 & 4.1 & - & $3-4$ & 4 & 13 \\
\hline 8 & $43 / \mathrm{F}$ & 13.9 & 4.3 & - & 4 & 4 & 10 \\
\hline 9 right & $56 / \mathrm{F}$ & 12.8 & 3.7 & - & $3-4$ & 3 & 6 \\
\hline 9 left & & 10.2 & 2.8 & 4 & $3-4$ & 3 & 6 \\
\hline 10 & $43 / \mathrm{F}$ & 10.5 & 2.9 & - & 3 & 4 & 7 \\
\hline 11 & $65 / \mathrm{F}$ & 15.2 & 5.2 & - & 4 & 3 & 8 \\
\hline
\end{tabular}




\begin{tabular}{lcccc}
\multicolumn{5}{c}{ Table 2} \\
The VCSS scores of patients \\
Patient/side & \multicolumn{5}{c}{ VCSS scores } \\
\cline { 2 - 5 } 1 right & Pre-procedural & First month & Sixth month & Twelve month \\
1 left & 7 & 2 & 2 & 1 \\
2 & 7 & 2 & 2 & 1 \\
3 & 6 & 0 & 0 & 0 \\
4 & 6 & 1 & 1 & 1 \\
5 & 7 & 1 & 1 & 1 \\
6 & 6 & 0 & 0 & 0 \\
7 & 16 & 9 & 4 & 4 \\
8 & 13 & 8 & 4 & 1 \\
9 right & 10 & 2 & 2 & 0 \\
9 left & 6 & 0 & 0 & 0 \\
10 & 6 & 0 & 0 & 0 \\
11 & 7 & 1 & 1 & 0 \\
\hline VCsS: Venous Clinical Severity Score. & & 0 & 0 & \\
\hline
\end{tabular}

Patient 7 had Cockett insufficiency in both pre- and post-procedural period.

There were no mortality and morbidity related to the procedure. No ecchymosis, skin pigmentation, hematoma, paresthesia, deep venous thromboembolism, or pulmonary embolism were observed. The mean VCSS of the patients was $8.1 \pm 3.1$ (range, 6.0 to 16.0 ) in the pre-procedural period and $1.1 \pm 1.4$ (range, 0.0 to 4.0 ) at 12 months of follow-up ( $\mathrm{p}=0.001)$. The pre- and post-procedural VCSS are given in Table 2. The mean diameter of deep veins was $13.0 \pm 1.8$ (range, 10.2 to 16.1 ) $\mathrm{mm}$ in the pre-procedural period and $8.9 \pm 1.2$ (range, 7.5 to 11.0) $\mathrm{mm}$ at the post-procedural 12 months.

\section{DISCUSSION}

In this study, we examined the efficacy of ICT in the treatment of lower limbs for primary DVI. Our results showed that the DVI and VCSS of the patients significantly improved at 12 months of follow-up. No morbidity and mortality were observed in any patients.

Although the application of this procedure has been increasing recently, the number of studies reported in the literature is still very small. In a study performed by Yavuz et al., ${ }^{[10]} 43$ patients were treated and the mean VCSS score improved from $20.7 \pm 5.9$ to $3.9 \pm 0.9$ at 12 months of follow-up. The mean deep vein vessel diameter at the level of the valve was $12.4 \pm 2.6 \mathrm{~mm}$ in the pre-procedural period, it improved to $9.0 \pm 1.7 \mathrm{~mm}$ after the procedure. Consistent with this study, the patients included in our study were successfully treated for DVI.

The ICT has certain advantages relative to treatment of DVI with conventional surgical approaches, such as valve reconstruction. After the conventional surgical methods, there is a risk of DVT due to venotomy and the need for anticoagulant treatment to avoid this and, also, the risk of surgical infection and the need for antibiotic prophylaxis to avoid this, in addition to cosmetic concerns. ${ }^{[5,7]}$ Postoperative bleeding, hematoma, and longer hospital stay are the other disadvantages of valve reconstructions. In one study, the mean duration of hospitalization in the postoperative period was five days in conventional reconstructive surgery. ${ }^{[5]}$ However, patients in whom ICT is performed can be often discharged on the same day. In our study, no postoperative adverse conditions were observed, and all patients were discharged on the same day after the procedure. No antibiotherapy or anticoagulant therapy was recommended to the patients.

Currently, valve reconstruction surgery for DVI is mainly performed to provide regression of ulcers 
in CEAP Class 5-6 patients. ${ }^{[9]}$ Considering the success and complications of conventional surgery, it is understandable why it is not routinely performed in every center. Also, ICT can be applied to patients with CEAP Class 3-4. ${ }^{[10]}$ Before complications occur, such as perimalleolar ulcer, it can be performed to ensure symptomatic clinical improvement. In our study, the CEAP classification of our patient population was mostly Class 3 and 4, only one patient had perimalleolar ulcer secondary to venous insufficiency. At three months of follow-up, the ulcer healed. In addition, a considerable improvement was observed in the VCSS at 12 months compared to the pre-procedural period. At 12 months of follow-up, as the insufficiency continued along the VSM in Patient 6 and Cockett vein insufficiency persisted in Patient 7, VCSS of these patients improved up to 4 .

In the current guidelines, symptomatic venous insufficiency is primarily recommended to be treated for superficial and perforating vein insufficiency. If advanced venous insufficiency (CEAP Class $\geq 4 \mathrm{~b}$ ) continues despite interventional therapies and compression treatments for superficial venous insufficiency, valve incompetency reconstruction surgery is planned for DVI..$^{[4,9,11,12]}$ However, in the prospective, randomized study of Wang et al., ${ }^{[8]}$ valvuloplasty for DVI and superficial venous insufficiency treatment were more beneficial than only superficial venous insufficiency treatment in terms of the healing of venous insufficiency and related ulcers. Hardy et al. ${ }^{[13]}$ and Tripathi et al. ${ }^{[14]}$ also suggested that valve reconstructions should be performed for DVI together with superficial vein surgery. In our study, although the symptoms regressed with ICT alone in Patient 6, complete treatment was not provided. In Patient 9, intravenous cyanoacrylate was first applied to the saphenous vein for venous insufficiency in the second leg. However, since DVI did not improve after the procedure, ICT was applied to this leg as well, and the symptoms then improved. Similarly, in the study of Makarova et al., ${ }^{[15]}$ reflux in the femoral vein did not improve with superficial vein surgery. Considering all these findings, our study results indicate that ICT can be performed as a priority, as the procedure is minimally invasive under local anesthesia and independent from superficial and perforating venous insufficiency surgery. As a matter of fact, in our study, Patients 2 and 5 had proximal VSM insufficiency and we treated them with only ICT. Their complaints improved in the post-procedureal period, although proximal VSM insufficiency was not interfered. As in the work of Eberhardt and Raffetto, ${ }^{[2]}$ we suggest that ICT can be considered primarily in patients with proximal superficial venous insufficiency and DVI, considering that some of the superficial insufficiency may result from DVI. In addition, since the proximal VSM diameter enlarged to 6.0 and $6.9 \mathrm{~mm}$, respectively in Patients 3 and 4 with proximal VSM insufficiency in the pre-procedural period, the leakage was reduced by decreasing the diameter of the VSM in this area by simultaneously delivering the ICT hard gel around the SFJ. Although ICT was performed around the common femoral vein in all patients included in our study, the ICT technique was observed to be a new and improved technique according to the results of these last two patients. We consider that CDUS-guided intervention in the treatment of primary DVI can be performed not only in the region where the common femoral vein-SFJ joins, but also in different segments where the structure and function of the venous valves of the lower extremity are normal. It can be considered that superficial venous insufficiency as well as DVI can be treated with ICT application from multiple levels of subcutaneous tissue into the CDUS-guided paravalvular area. Undoubtedly, we believe that it would contribute to the extension of ICT application indication in selected cases without advanced tortuosity, thrombophlebitis history, postphlebitic syndrome, or venous packaging.

Nonetheless, there may be certain difficulties in implementing ICT. This therapy can be performed by vascular surgeons with a very good CDUS dominance. The common femoral artery, vein, and nerves are very close to each other anatomically in this region, as well as bifurcation of the deep femoral artery and vein bifurcation in this area are anatomical conditions which increase the difficulty of the procedure. Both axial section and long axis images of deep venous structures and paravalvular area should be well evaluated with CDUS before and during the procedure to avoid complications and to achieve successful results. Otherwise, complications such as potential hematoma, vascular injury, venous valve injury, arterial pseudoaneurysm development, iatrogenic arteriovenous fistula formation, and femoral nerve injury may be inevitable. In addition, if the ICT hard gel is delivered intravenously by mistake, deep venous adhesion and obstruction, and pulmonary embolism can occur. On the other hand, allergic reactions may rarely occur in patients against cyanoacrylate. ${ }^{[16,17]}$ 
No evidence of these complications was observed in our patient population. Toxicological, carcinogenic, and mutagenic effects against hyaluronic acid and NBCA have not been observed in vascular use until today. ${ }^{[13,18,19]}$

The present study has some limitations. The major limitation is its retrospective design with a limited sample size. The retrospective nature of the study precludes the elimination of all potential confounders and biases. Its single-center design conducted by a single specialist for selected patients is another limitation. Nevertheless, in the current literature, there is only one study about the results of this treatment method, as it is a fairly new method. Therefore, we believe that the results of our study would contribute additional information to body of knowledge in the current literature.

In conclusion, internal compression therapy procedure appears to be promising, since it is minimally invasive, rapid, and effective method for the treatment of patients with deep venous insufficiency as an alternative treatment modality of conventional valve reconstruction surgery. In addition, this procedure can be applied under outpatient conditions due to the ease of application, postoperative early recovery, and satisfactory cosmetic results. For internal compression therapy applications in deep and superficial venous insufficiency, further large-scale, prospective, randomized studies are needed.

\section{Declaration of conflicting interests}

The authors declared no conflicts of interest with respect to the authorship and/or publication of this article.

\section{Funding}

The authors received no financial support for the research and/or authorship of this article.

\section{REFERENCES}

1. Wittens C, Davies AH, Bækgaard N, Broholm R, Cavezzi A, Chastanet S, et al. Editor's Choice - Management of Chronic Venous Disease: Clinical Practice Guidelines of the European Society for Vascular Surgery (ESVS). Eur J Vasc Endovasc Surg 2015;49:678-737.

2. Eberhardt RT, Raffetto JD. Chronic venous insufficiency. Circulation 2014;130:333-46.

3. Doliner B, Jaller JA, Lopez AJ, Lev-Tov H. Treatments to prevent primary venous ulceration after deep venous thrombosis. J Vasc Surg Venous Lymphat Disord 2019;7:260-71.e1.
4. Leckie KE, Dalsing MC. Open Surgical Reconstruction for Deep Venous Occlusion and Valvular Incompetence. Surg Clin North Am 2018;98:373-84.

5. Maleti O, Lugli M, Perrin M. After superficial ablation for superficial reflux associated with primary deep axial reflux, can variable outcomes be caused by deep venous valve anomalies? Eur J Vasc Endovasc Surg 2017;53:229-36.

6. Maleti O, Lugli M, Tripathi RK. Deep venous reconstructive surgery. Semin Vasc Surg 2015;28:39-46.

7. Maleti O, Perrin M. Reconstructive surgery for deep vein reflux in the lower limbs: techniques, results and indications. Eur J Vasc Endovasc Surg 2011;41:837-48.

8. Wang SM, Hu ZJ, Li SQ, Huang XL, Ye CS. Effect of external valvuloplasty of the deep vein in the treatment of chronic venous insufficiency of the lower extremity. J Vasc Surg 2006;44:1296-300.

9. Maleti O, Lugli M. Valve reconstruction for deep venous reflux. In: Ochoa Chaar CI, editor. Current Management of Venous Diseases. 1st ed. New York: Springer; 2017. p. 217-29.

10. Yavuz T, Acar AN, Yavuz K, Ekingen E. A Retrospective Study of Deep Vein Insufficiency Treatment Device: ICT. Ann Vasc Dis Vol. 13, No. 3; 2020;13:1-6.

11. O'Donnell TF Jr, Passman MA, Marston WA, Ennis WJ, Dalsing M, Kistner RL, et al. Management of venous leg ulcers: clinical practice guidelines of the Society for Vascular Surgery ${ }^{\oplus}$ and the American Venous Forum. J Vasc Surg 2014;60(2 Suppl):3S-59S.

12. Ting AC, Cheng SW, Wu LL, Cheung GC. Changes in venous hemodynamics after superficial vein surgery for mixed superficial and deep venous insufficiency. World J Surg 2001;25:122-5.

13. Hardy SC, Riding G, Abidia A. Surgery for deep venous incompetence. Cochrane Database Syst Rev 2004;3:CD001097.

14. Tripathi R, Sieunarine K, Abbas M, Durrani N. Deep venous valve reconstruction for non-healing leg ulcers: techniques and results. ANZ J Surg 2004;74:34-9.

15. Makarova NP, Lurie F, Hmelniker SM. Does surgical correction of the superficial femoral vein valve change the course of varicose disease? J Vasc Surg 2001;33:361-8.

16. Lawson J, Gauw S, van Vlijmen C, Pronk P, Gaastra M, Mooij M, et al. Sapheon: the solution? Phlebology 2013;28 Suppl 1:2-9.

17. Gibson K, Ferris B. Cyanoacrylate closure of incompetent great, small and accessory saphenous veins without the use of post-procedure compression: Initial outcomes of a post-market evaluation of the VenaSeal System (the WAVES Study). Vascular 2017;25:149-56.

18. Linfante I, Wakhloo AK. Brain aneurysms and arteriovenous malformations: advancements and emerging treatments in endovascular embolization. Stroke 2007;38:1411-7.

19. Ragg JC, Despa OR, Brüggemann U, El-Chamali S, Stoyanova K. Foam sclerotherapy of the saphenous vein with adjuvant hyaluronan compression. Phlebologie 2017;46:81-6. 